\title{
ANNALES
}

POLONICI MATHEMATICI

$\mathrm{XV}$ (1964)

\section{A solution of the problem of four limits}

\author{
by W. BACH (Kraków)
}

\section{The formulation of the problem. The ecart and the} capacity of the set. F. Leja posed in 1938 in Annales de la Soc. Pol. de Math. 17 (1938), p. 130, the following problem ( $\left.{ }^{1}\right)$.

Let $R$ be the complex plane and $p^{(n)}=\left\{p_{0}, p_{1}, \ldots, p_{n}\right\}$ an arbitrary system of $n+1$ different points of a closed and bounded set $E$ and $u_{j k}=u_{j k}\left(z, p^{(n)}\right)$ the function defined by the formulas:

$$
u_{j k}=\frac{z-p_{k}}{p_{j}-p_{k}} \quad \text { for } \quad j \neq k, \quad u_{j i}=1 .
$$

Let us denote by $A_{n}(z), B_{n}(z), C_{n}(z), D_{n}(z)$, the greatest lower bounds of the products

$$
\begin{aligned}
A_{n}\left(z ; p^{(n)}\right) & =\prod_{j=0}^{n} \prod_{k=0}^{n}\left|u_{j k}\right|, \quad B_{n}\left(z ; p^{(n)}\right)=\max _{(i)} \prod_{k=0}^{n}\left|u_{j k} u_{k j}\right|, \\
C_{n}\left(z ; p^{(n)}\right) & =\max _{(j)} \prod_{k=0}^{n}\left|u_{j k}\right|, \quad D_{n}\left(z ; p^{(n)}\right)=\max _{(i)} \prod_{k=0}^{n}\left|u_{k j}\right|
\end{aligned}
$$

respectively, when the system $p^{(n)}$ changes in $E$.

It is known [1], [2], [3] that, if the ecart $v(E,|p-q|)$ of the set $E$ is positive $\left(^{2}\right)$, then the sequences $A_{n}(z)^{1 / n(n+1)}, B_{n}(z)^{1 / 2 n}, C_{n}(z)^{1 / n}, D_{n}(z)^{1 / n}$ are convergent to $A(z), B(z), C(z), D(z)$ respectively and that $A(z) \equiv B(z)$. Prove that $A(z) \equiv B(z) \equiv C(z) \equiv D(z)$ or that at least one of the equalities is false.

The object of the present paper is to give a solution of the above problem.

To begin with we shall introduce some definitions.

Let $\omega(p, q)$ be an arbitrary generating function, i.e. a function defined and continuous for every pair of points $p$ and $q$ of the complex plane and satisfing the conditions

$$
\omega(p, q) \geqslant 0, \quad \omega(p, q)=\omega(q, p)
$$

(1) See also Colloquium Mathematicum 7 (1959), p. 151.

(2) The ecart will be detined later. 
and let $E$ be a closed and bounded set in the complex plane. Let us consider a system $p^{(n)}=\left\{p_{0}, p_{1}, \ldots, p_{n}\right\}$ of $n+1$ points of $E$. Let us denote by $\vee\left(p^{(n)}\right)$ the product $\prod_{0 \leqslant j<k \leqslant n} \omega\left(p_{i}, p_{k}\right)$ and by $V_{n}(E, \omega)$ the supremum of the expression $\left.\mid \prod_{0 \leqslant j<k \leqslant n} \omega\left(p_{i}, p_{k}\right)\right]^{2 / n(n+1)}$ when $p^{(n)}$ changes arbitrarily in $E$. Since $E$ is closed and bounded and $\omega(p, q)$ is continuous, there exists a system

$$
q^{(n)}=\left\{q_{0}^{(n)}, q_{1}^{(n)}, \ldots, q_{n}^{(n)}\right\}
$$

which will also be denoted by $\left\{q_{0}, q_{1}, \ldots, q_{n}\right\}$, such that

$$
V_{n}(E, \omega)=\left[\vee\left(q^{(n)}\right)\right]^{2 / n(n+1)} .
$$

It is known [4] that there exists a finite limit

$$
\lim _{n \rightarrow \infty} V_{n}(E, \omega)=V(E, \omega) .
$$

System (1.1) will be called the $n$-th extremal system of $E$ with respect to (1) and the limit (1.2) will be called the ecart of $E$ with respect to $\omega$.

Let us denote by $I I$ the class of all non-negative Radon measures $\mu(e)$ defined on the subsets of $E$ and satisfing the condition $\mu(E)=1$.

Let $\delta$ be an arbitrary positive number. Let us denote by $\omega_{\delta}(p, q)$ the function defined as follows:

$$
\omega_{\delta}(p, q)= \begin{cases}\omega(p, q) & \text { for } \quad \omega(p, q) \geqslant \delta \\ \delta & \text { for } \quad \omega(p, q)<\delta .\end{cases}
$$

For fixed $\delta$ the function $\log \frac{1}{\omega_{\delta}(p, q)}$ is continuous with respect to the pair of points $p$ and $q$ and non-decreasing with respect to $\delta$. Then the integrals

$$
\int_{E} \log \frac{1}{\omega_{\delta}(p, q)} d \mu(q), \quad \int_{E}\left[\int_{E} \log \frac{1}{\omega_{\delta}(p, q)} d \mu(q)\right] d \mu(p)
$$

and their limits (finite or not) as $\delta \rightarrow 0$ exist. The limit of the first integral will be denoted by

$$
\int_{E} \log \frac{1}{\omega(p, q)} d \mu(q)
$$

and the limit of the second integral by

$$
\int_{E}\left[\int_{E} \log \frac{1}{\omega(p, q)} d \mu(q)\right] d \mu(p),
$$

or shortly by $I(\mu)$. 
Let $K=\max \left(1, \sup _{p, q \in E} \omega(p, q)\right)$. Since $E$ is a closed and bounded set and $\omega(p, q)$ is continuous, $K$ is a finite number $\geqslant 1$. Since $\log \frac{K}{\omega(p, q)} \geqslant 0$ (in the case where $\omega(p, q)=0$ we put $\log \frac{K}{\omega(p, q)}=\infty$ ) and since

$$
\int_{E}\left[\int_{E} \log \frac{1}{\omega(p, q)} d \mu(q)\right] d \mu(p)-\log \frac{1}{K}=\int_{E}\left[\int_{E} \log \frac{K}{\omega(p, q)} d \mu(q)\right] d \mu(p)
$$

we have

$$
\int_{E}\left[\int_{E} \log \frac{1}{\omega(p, q)} d \mu(q)\right] d \mu(p) \geqslant \log \frac{1}{K} .
$$

We shall consider two cases:

Case 1. There exists a $\mu \in M$ such that $I(\mu)<\infty$. In this case we can prove by the method as in [1] that there exists an $\eta \in I$ such that

$$
I(\eta)=\inf _{\mu \in M I} I(\mu)
$$

By (1.3) we have $I(\eta)>-\infty$. The measure $\eta(e)$ will be called the equilibrium measure with respect to the function $\omega$. The function $U(p)$ defined in the whole plane by the formula

$$
U(p)=\int_{E} \log \frac{1}{\omega(p, q)} d \eta(q)
$$

will be called the equilibrium potential with respect to $\omega$ and the number

$$
C(E, \omega)=e^{-I(\eta)}
$$

the capacity of the set $E$ with respect $\omega$.

Case 2. $I(\mu)=\infty$ for every $\mu \in M$. In this case we put $C(E, \omega)=0$. In this case we do not define the equilibrium measure and the equilibrium potential.

\section{The equality of the ecart and the capacity of a set.}

Lemma 1. For an arbitrary closed and bounded set $E$ in the plane and for an arbitrary generating function $\omega(p, q)$ we have

$$
C(E, \omega)=V(E, \omega) .
$$

Proof. Let $\delta$ be an arbitrary positive number and $\omega_{\delta}(p, q)$ a function defined on page 58. Let $V_{n}\left(E, \omega_{\delta}\right), V\left(E, \omega_{\delta}\right)$ and $C\left(E, \omega_{\delta}\right)$ be defined analogically to $V_{n}(E, \omega), V(E, \omega)$ and $C(E, \omega)$. It is sufficient to prove that

$$
\begin{gathered}
V\left(E, \omega_{\delta}\right)=C\left(E, \omega_{\delta}\right), \\
V\left(E, \omega_{\delta}\right) \rightarrow V(E, \omega) \text { as } \quad \delta \rightarrow 0, \\
C\left(E, \omega_{\delta}\right) \rightarrow C(E, \omega) \text { as } \quad \delta \rightarrow 0 .
\end{gathered}
$$


We shall first prove (2.1). Let $K=\sup _{p, q \in E} \omega_{\delta}(p, q)$. Since $E$ is closed and bounded and $\omega_{\delta}(p, q)$ is continuous, $K$ is a finite number $\geqslant \delta$. Let $\omega_{\delta}^{1}(p, q)=\omega_{\delta}(p, q) / K$. It is easy to see that $\omega_{\delta}^{1}(p, q) \leqslant 1$ for $p, q \in E$ and $V\left(E, \omega_{\delta}^{1}\right)=V\left(E, \omega_{\delta}\right) / K, C\left(E, \omega_{\delta}^{1}\right)=C\left(E, \omega_{\delta}\right) / K$. In order to prove $(2.1)$ it is sufficient to prove that $C\left(E, \omega_{\delta}^{1}\right)=V\left(E, \omega_{\delta}^{1}\right)$.

In the sequel we shall write $\omega_{\delta}(p, q)$ instead of $\omega_{\delta}^{1}(p, q)$. Hence we have $\omega_{\mathcal{S}}(p, q) \leqslant 1$ for $p, q \in E$.

Let $\mu(e)$ be a measure belonging to $M$ (see p. 58). Let us denote by $I^{\delta}(\mu)$ the integral defined by the formula

$$
I^{\delta}(\mu) \stackrel{\mathrm{df}}{=} \int_{E}\left[\int_{E} \log \frac{1}{\omega_{\delta}(p, q)} d \mu(q)\right] d \mu(p)
$$

and by $\eta_{\delta}(e)$ a measure realising the greatest lower bound of the integrals $I^{s}(\mu)$ when $\mu \in M$ :

$$
I^{\delta}\left(\eta_{\delta}\right)=\inf _{\mu \in M} I(\mu)
$$

Without any loss of generality we can assume that there exists a number $a>1$ such that $E$ is contained in the rectangle

$$
0 \leqslant x \leqslant a-1, \quad 0 \leqslant y \leqslant a-1 .
$$

Let us divide the square $0 \leqslant x<a, 0 \leqslant y<a$ into $n^{2}$ partially open squares

$$
\frac{j-1}{n} a \leqslant x<\frac{j}{n} a, \quad \frac{k-1}{n} a \leqslant y<\frac{k}{n} a, \quad j, k=1,2, \ldots, n
$$

and let us denote these squares by $P_{i}^{(n)}, i=1,2, \ldots, n^{2}$. Let us denote the sets $E \cap P_{i}^{(n)}$ by $E_{i}^{(n)}$ for $i=1,2, \ldots, n^{2}$. Hence we have

$$
\sum_{i=1}^{n^{2}} E_{i}^{(n)}=E
$$

Let $m(n)$ be a natural number and $l_{i}(n), i=1,2, \ldots, n^{2}$ non-negative integers such that

$$
\frac{l_{i}(n)}{m(n)}-\frac{1}{n^{5}} \leqslant \eta_{\delta}\left(E_{i}^{(n)}\right) \leqslant \frac{l_{i}(n)}{m(n)}, \quad i=1,2, \ldots, n^{2} .
$$

If the set $E_{i}^{(n)}$ is empty we put $l_{i}(n)=0$. We can assume (taking suitable $m(n))$ that

$$
\sum_{i=1}^{n^{2}} l_{i}(n) \rightarrow \infty, \quad \text { as } \quad n \rightarrow \infty
$$


Let us take $l_{i}(n)$ arbitrary points (different or not) in every set $E_{i}^{(n)}$ $\left(i=1,2, \ldots, n^{2}\right)$. We shall have the system of the $l(n)$ points

$$
\left\{p_{0}, p_{1}, \ldots, p_{l(n)}\right\} \quad \text { where } \quad l(n)=\sum_{i=1}^{n^{2}} l_{i}(n)-1 .
$$

Next, for brevity we shall denote the sets $E_{i}^{(n)}$ by $E_{i}$ and the numbers $l(n), l_{i}(n), m(n)$ by $l, l_{i}, m$.

Let

$$
\left\{q_{0}, q_{1}, \ldots, q_{l}\right\}
$$

be the $l$ th extremal system of the set $E$ with respect to the function $\omega_{\delta}$. Let us denote by $\mu_{l}(e)$ and $\mu_{l}^{\prime}(e)$ the measures defined on the Borel subsets $e$ of $E$ as follows:

$$
\mu_{l}(e)=\frac{k}{l+1}, \quad \mu_{l}^{\prime}(e)=\frac{k^{\prime}}{l+1},
$$

where $k$ and $k^{\prime}$ denote the numbers of the points of system (2.5) and of the extremal system (2.6) contained in $e$. It is easy to see that the measures $\mu_{l}$ and $\mu_{l}^{\prime}$ belong to the class $M$ (see p. 58). From the definition of the extremal system (2.6) we have

$$
\begin{aligned}
& \log \frac{1}{V_{l}\left(E, \omega_{\delta}\right)}=\frac{2}{l(l+1)} \sum_{0 \leqslant i<k \leqslant l} \log \frac{1}{\omega_{\delta}\left(q_{i}, q_{k}\right)} \\
& =\frac{l+1}{l}\left[\frac{1}{(l+1)^{2}} \sum_{i, k=0}^{l} \log \frac{1}{\omega_{\delta}\left(q_{i}, q_{k}\right)}-\frac{1}{(l+1)^{2}} \sum_{i=0}^{l} \log \frac{1}{\omega_{\delta}\left(q_{i}, q_{i}\right)}\right] .
\end{aligned}
$$

Since

$$
\frac{1}{(l+1)^{2}} \sum_{i, k=0}^{l} \log \frac{1}{\omega_{\delta}\left(q_{i}, q_{k}\right)}=\int_{E}\left[\int_{E} \log \frac{1}{\omega_{\delta}(p, q)} d \mu_{l}(q)\right] d \mu_{l}(p) \stackrel{\mathrm{d} f}{=} I^{\delta}\left(\mu_{l}\right)
$$

and

$$
\log \frac{1}{\omega_{\delta}(p, q)} \leqslant \log \frac{1}{\delta}
$$

we have from (2.7) and from the definition of $\mu_{l}$ and $\eta_{\delta}$

(2.8) $\log _{\overline{V_{l}}\left(\bar{E}, \overline{\omega_{\delta}}\right.} \geqslant \frac{l+1}{l}\left[I^{\delta}\left(\mu_{l}\right)-\frac{1}{l+1} \log \frac{1}{\delta}\right] \geqslant \frac{l+1}{l}\left[I^{\delta}\left(\eta_{\delta}\right)-\frac{1}{l+1} \log \frac{1}{\delta}\right]$.

Since $V_{l}\left(E, \omega_{\delta}\right) \rightarrow V\left(E, \omega_{\delta}\right)$ as $l \rightarrow \infty$ (p. 58), we shall obtain from (2.8) the inequality

$$
\log \frac{1}{V\left(E, \omega_{\delta}\right)} \geqslant I^{\delta}\left(\eta_{\delta}\right)
$$


and hence

$$
\Gamma\left(E, \omega_{\delta}\right) \leqslant \exp \left[-I^{\delta}\left(\eta_{0}\right)\right]=C\left(E, \omega_{\delta}\right)
$$

In order to prove $(2.1)$ it is sufficient to prove that $V\left(E, \omega_{\delta}\right)$ $\geqslant C\left(E, \omega_{\delta}\right)$.

Since system (2.6) is extremal, we have

$$
\begin{aligned}
\log \frac{1}{V_{l}\left(E, \omega_{\delta}\right)} & =\frac{2}{l(l+1)} \sum_{0 \leqslant i<k \leqslant l}^{\top} \log \frac{1}{\omega_{\delta}\left(q_{i}, q_{k}\right)} \leqslant \frac{2}{l(l+1)} \sum_{0 \leqslant i<k \leqslant l} \log \frac{1}{\omega_{\delta}\left(p_{i}, p_{k}\right)} \\
& =\frac{l+1}{l}\left[\frac{1}{(l+1)^{2}} \sum_{i, k=0}^{l} \log \frac{1}{\omega_{\delta}\left(p_{i}, p_{k}\right)}-\frac{1}{(l+1)^{2}} \sum_{i=0}^{l} \log \frac{1}{\omega_{\delta}\left(p_{i}, p_{i}\right)}\right] .
\end{aligned}
$$

Let $M_{i k}=\sup _{p \in E_{i}, q \in E_{k}} \log \frac{1}{\omega_{\delta}(p, q)}$. Since

$$
\frac{1}{(l+1)^{2}} \sum_{i, k=0}^{l} \log \frac{1}{\omega_{\delta}\left(p_{i},\right.}, \overline{\left.p_{k}\right)}=I^{\delta}\left(\mu_{l}\right)
$$

we shall obtain from (2.9)

$$
\begin{aligned}
\log \frac{1}{V_{l}\left(E, \omega_{\delta}\right)} & \leqslant \frac{l+1}{l}-\left(I^{j}\left(\mu_{l}\right)-\frac{\log (1 / M)}{l+1}\right) \\
& \leqslant \frac{l+1}{l}\left(\sum_{i, k=1}^{n^{2}} M_{i k} \mu_{l}\left(E_{i}\right) \mu_{l}\left(E_{k}\right)-\frac{\log (1 / M)}{l+1}\right)
\end{aligned}
$$

where $M=\sup _{p, q \in E} \log \frac{1}{\omega_{d}(p, q)}$. From inequality $(2.4)$ and from the definition of $\mu_{l}$ we have

$$
\sum_{i, k=1}^{n^{2}} M_{i k} \mu_{l}\left(E_{i}\right) \mu_{l}\left(E_{k}\right)=\sum_{i, k=1}^{n^{2}} M_{i k} \frac{l_{i}}{m} \cdot \frac{l_{k}}{m} \leqslant \sum_{i, k=1}^{n^{2}} M_{i k}\left[\eta_{\delta}\left(E_{i}\right)+\frac{1}{n^{5}}\right]\left[\eta_{\delta}\left(E_{k}\right)+\frac{1}{n^{5}}\right]
$$

and hence

$$
\begin{aligned}
\log \frac{1}{V_{l}\left(E, \frac{\left.\omega_{\delta}\right)}{\omega} \leqslant \frac{l+1}{l}\right.}\left[\sum_{i, k=1}^{n^{2}} M_{i k} \eta_{\delta}\left(E_{i}\right) \eta_{\delta}\left(E_{k}\right)+\frac{1}{n^{10}} \sum_{i, k=1}^{n^{2}} M_{i k}+\right. \\
\left.+\frac{1}{n^{5}} \sum_{i, k=1}^{n^{2}} M_{i k} \eta_{\delta}\left(E_{i}\right)+\frac{1}{n^{5}} \sum_{i, k=1}^{n^{2}} M_{i k} \eta_{\delta}\left(E_{k}\right)-\frac{\log (1 / M)}{l+1}\right]
\end{aligned}
$$


Since $M_{i k} \leqslant \log (1 / \delta)$ and $0 \leqslant \eta_{\delta}\left(E_{i}\right) \leqslant 1$, we have from the last inequality

(2.10) $\quad \log \frac{1}{V_{l}(E, \omega)}$

$$
\leqslant \frac{l+1}{l}\left[\sum_{i, k=1}^{n^{2}} M_{i k} \eta_{\diamond}\left(E_{i}\right) \eta_{\diamond}\left(E_{k}\right)+\frac{\log (1 / \delta)}{n^{6}}+\frac{2 \log (1 / \delta)}{n}-\frac{\log (1 / M)}{l+1}\right] .
$$

The function $\log \frac{1}{\omega_{\delta}(p, q)}$ is uniformly continuous in the set $E \times E$; so for any $\varepsilon>0$ there exists a $N(\varepsilon)$ such that

$$
M_{i k}-\log \frac{1}{\omega_{\delta}(p, q)}<\varepsilon \quad \text { for } \quad p \in E_{i}, q \in E_{k}, n \geqslant N(\varepsilon) .
$$

Hence

$$
\begin{aligned}
\sum_{i, k=1}^{n^{2}} M_{i k} \eta_{\delta}\left(E_{i}\right) \eta_{\delta}\left(E_{k}\right)= & \sum_{i, k=1}^{n^{2}} \int_{E_{i}}\left[\int_{E_{k}} M_{i k} d \eta_{\delta}(q)\right] d \eta_{\delta}(p) \\
\leqslant & \sum_{i, k=1}^{n^{2}} \int_{E_{i}}\left[\int_{E_{k}} \log \frac{1}{\omega_{\delta}(p, q)} d \eta_{\delta}(q)\right] d \eta_{\delta}(p)+ \\
& +\varepsilon \sum_{i, k=1}^{n^{2}} \int_{E_{i}}\left[\int_{E_{k}} d \eta_{\delta}(q)\right] d \eta_{\delta}(p)=I^{\delta}\left(\eta_{\delta}\right)+\varepsilon
\end{aligned}
$$

and from (2.10) we have

$$
\log \frac{1}{V_{l}\left(E, \omega_{\delta}\right)} \leqslant I^{\delta}\left(\eta_{\delta}\right)+\varepsilon+\frac{\log (1 / \delta)}{n^{8}}+\frac{2 \log (1 / \delta)}{n}-\frac{\log (1 / M)}{l+1} .
$$

Since $l=l(n) \rightarrow \infty$ as $n \rightarrow \infty$, we have from the last inequality

$$
\log \frac{1}{V\left(E, \omega_{\delta}\right)} \leqslant I^{\delta}\left(\eta_{\delta}\right)+\varepsilon .
$$

Since $\varepsilon$ is an arbitrary positive number, we shall hence obtain

or

$$
\log \frac{1}{V\left(E, \omega_{\delta}\right)} \leqslant I^{\delta}\left(\eta_{\delta}\right)
$$

$$
V\left(E, \omega_{\delta}\right) \geqslant \exp \left[-I^{\delta}\left(\eta_{\delta}\right)\right]=C\left(E, \omega_{\delta}\right) .
$$

Hence and from the opposite inequality (see p. 62) we have equality (2.1).

Proof of (2.2). It is known [5] that

$$
V_{n+1}^{r}(E, \omega) \leqslant V_{n}(E, \omega) \quad \text { for } \quad n=1,2, \ldots
$$


From the definition of $\omega_{\delta}$ it follows that

$$
V_{n}\left(E, \omega_{\jmath}\right) \geqslant V_{n}(E, \omega), \quad n=1,2, \ldots
$$

Hence and from formula (1.2) (see p. 58) follows

$$
V\left(E, \omega_{\delta}\right) \geqslant V(E, \omega)
$$

In order to prove $(2.2)$ it is sufficient to prove that for any $\varepsilon>0$ there exists a $\delta_{0}(\varepsilon)$ such that

$$
V\left(E, \omega_{\delta}\right)<V(E, \omega)+\varepsilon \quad \text { for } \quad \delta<\delta_{0}=\delta_{0}(\varepsilon) .
$$

Since $V_{n}(E, \omega) \rightarrow V(E, \omega)$ there exists a natural number $N(\varepsilon)$ such that

$$
V_{n}(E, \omega) \leqslant V(E, \omega)+\varepsilon / 2 \quad \text { for } \quad n \geqslant N(\varepsilon)
$$

Next we shall prove that for $N=N(\varepsilon)$ there exists a $\delta_{0}$ such that

$$
V_{N}\left(E, \omega^{\delta}\right)<V_{N}(E, \omega)+\varepsilon / 2 \quad \text { for } \quad \delta<\delta_{0} .
$$

Let $q_{0}, q_{1}, \ldots, q_{N}$ be the $N$ th extremal system of $E$ with respect to $\omega_{0}$. If $V_{N}(E, \omega)>0$ we have the equality

$$
\omega_{\delta}\left(q_{i}, q_{k}\right)=\omega\left(q_{i}, q_{k}\right)
$$

for

$$
\delta<\delta_{0}=\min \left(K, \frac{\left[V_{N}(E, \omega)\right]}{K \frac{\frac{N(N+1)}{2}}{K^{\frac{N(N+1)}{2}}-1}}\right), \quad K=\sup _{p, q \in E} \omega_{\delta}(p, q)
$$

because in the contrary case there would exist a positive number $\delta<\delta_{0}$ and a pair of points $q_{i_{0}}, q_{k_{0}}, 0 \leqslant i_{0} \leqslant N, 0 \leqslant k_{0} \leqslant N$ such that $\omega_{\delta}\left(q_{i_{0}}, q_{k_{0}}\right)$ $=\delta<\delta_{0}$. From the definition of $K$ and $\delta$ we should have

$$
\nabla_{N}\left(E, \omega_{\delta}\right)=\left[\prod_{0 \leqslant i<k \leqslant N} \omega_{\delta}\left(q_{i}, q_{k}\right)\right]^{\frac{2}{N(N+1)}} \leqslant\left[\delta K^{\frac{N(N+1)}{2}-1}\right]^{\frac{2}{N(N+1)}} \leqslant \nabla_{N}(E, \omega),
$$

which is absurd. Hence

$$
\begin{aligned}
V_{N}\left(E, \omega_{\delta}\right) & =\left[\prod_{0 \leqslant i<k \leqslant N} \omega_{\delta}\left(q_{i}, q_{k}\right)\right]^{\frac{2}{N(N+1)}}=\left[\prod_{0 \leqslant i<k \leqslant N} \omega\left(q_{i}, q_{k}\right)\right]^{\frac{2}{N(N+1)}} \\
& <V_{N}(E, \omega)+\varepsilon / 2
\end{aligned}
$$

i.e. we have inequality (2.15). 


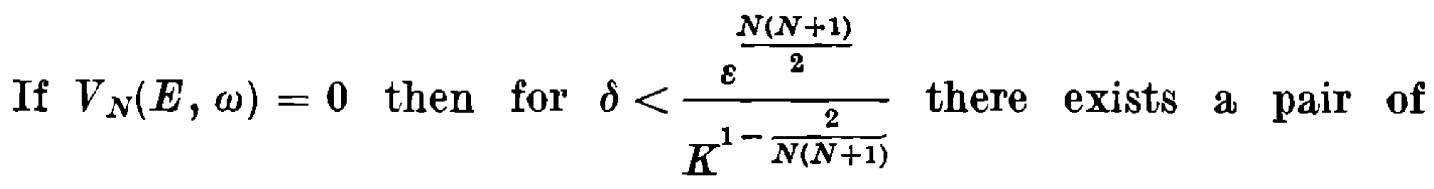
points $q_{i_{1}}, q_{k_{1}}$ of the extremal system such that $\omega_{\delta}\left(q_{i_{1}}, q_{k_{1}}\right)=\delta$; so

$$
V_{N}\left(E, \omega_{\delta}\right) \leqslant\left[\delta K^{\frac{N(N+1)}{2}-1}\right] \frac{2}{N(N+1)}<\varepsilon / 2=V_{N}(E, \omega)+\varepsilon / 2
$$

and inequality (2.15) is also proved.

From (2.14) and (2.15) it follows that

$$
V_{N}\left(E, \omega_{\delta}\right) \leqslant V(E, \omega)+\varepsilon \quad \text { for } \quad \delta<\delta_{0}
$$

and from (2.11) and (2.16) we have

$$
V\left(E, \omega_{\delta}\right)<V(E, \omega)+\varepsilon \quad \text { for } \delta<\delta_{0},
$$

i.e. inequality (2.13).

Since $\varepsilon$ is an arbitrary positive number, formula (2.2) follows hence.

Proof of (2.3). We shall consider two cases.

Case 1. $V(E, \omega)>0$. In this case from (2.1) and (2.2) it follows that

$$
C\left(E, \omega_{\delta}\right) \rightarrow V(E, \omega) \quad \text { as } \quad \delta \rightarrow 0 .
$$

Hence it follows that $I^{\delta}\left(\eta_{\delta}\right)$ converges to a finite limit $I$ as $\delta \rightarrow 0$ :

$$
\lim _{\delta \rightarrow 0} I^{\delta}\left(\eta_{\delta}\right)=I \text {. }
$$

From the definition of $\eta$ (see p. 59) and $\eta_{\delta}$ (see p. 60) we have

$$
I^{\delta}\left(\eta_{\delta}\right) \leqslant I^{\delta}(\eta)
$$

From (2.17), (2.18) and the definition of the integral $I(\eta)$ we obtain the inequality

$$
I \leqslant I(\eta)
$$

Now we shall prove that $I=I(\eta)$. In the opposite case there would exist a number $\varepsilon>0$ such that

$$
I<I(\eta)-\varepsilon
$$

Let $\left\{\delta_{n}\right\}$ be a sequence of positive numbers such that $\delta_{n} \rightarrow 0$ and $I^{\delta_{n}}\left(\eta_{\delta_{n}}\right) \rightarrow I$. We can suppose that the sequence of the measures $\eta_{\delta_{n}}(e)$ is convergent to a measure $\eta_{0}(e)$ (because from the sequence of such measures we can take a convergent subsequence, see [1]). Let $\varepsilon^{\prime}$ be an arbitrary positive number. It is easy to see that $I^{\delta_{n}}\left(\eta_{\delta_{n}}\right) \geqslant I^{\varepsilon^{\prime}}\left(\eta_{\delta_{n}}\right)$ for $\delta_{n}<\varepsilon^{\prime}$ and hence if $n \rightarrow \infty$, we shall obtain $I \geqslant I^{\varepsilon^{\prime}}\left(\eta_{0}\right)$. Now if $\varepsilon^{\prime} \rightarrow 0$ we shall have $I \geqslant I\left(\eta_{0}\right)$, which together with inequality $(2.20)$ gives the inequality $I\left(\eta_{0}\right)<I(\eta)-\varepsilon$ in contradiction to the definition of $\eta$. Therefore $I=I(\eta)$. 
Hence and from equality (2.1) we have

$$
C\left(E, \omega_{\delta}\right)=e^{-I^{\delta}\left(\eta_{\delta}\right)} \rightarrow e^{-I}=e^{-I(\eta)}=C(E, \omega) \quad \text { as } \quad \delta \rightarrow 0 .
$$

Case 2. $V(E, \omega)=0$. In this case from (2.1) and (2.2) it follows that $I^{\delta}\left(\eta_{\delta}\right) \rightarrow \infty$ as $\delta \rightarrow 0$. Hence and from the definition of $\eta_{\delta}$ it follows that $I^{\delta}(\mu) \rightarrow \infty$ as $\delta \rightarrow 0$ for $\mu \epsilon M$ and hence $I(\mu)=\infty$ for $\mu \epsilon M$. By the definition of capacity we have in this case $C(E, \omega)=0=V(E, \omega)$. Formula (2.3) is thus proved.

From (2.1), (2.2) and (2.3) it follows that $C(E, \omega)=V(E, \omega)$, q.e.d.

\section{The properties of the extremal measure and its con-} nection with the Green function. Let $E$ be a closed set on the $z$-plane and $z$ be a point not belonging to $E$. Suppose $C(E,|p-q|)>0$. Since (see p. 57)

$$
A_{n}\left(z ; p^{(n)}\right)=\prod_{\substack{j, k=0 \\ j \neq k}}^{n} \frac{\sqrt{\left|\bar{z}-p_{j}\right|\left|z-p_{k}\right|}}{\left|p_{j}-p_{k}\right|} \quad \text { and } \quad A_{n}(z)=\inf _{p^{(n)} \in E} A_{n}\left(z ; p^{(n)}\right)
$$

it follows that

$$
\log A_{n}(z)=\inf _{p^{(n)}} \log A_{n}(z)=2 \sum_{0 \leqslant j<k \leqslant n} \log \frac{1}{\omega\left(q_{i}, q_{k} ; z\right)},
$$

where $\omega(p, q ; z)=\frac{|p-q|}{\sqrt{|z-p||z-q|}}$ and $q_{0}, q_{1}, \ldots, q_{n}$ is the $n$th extremal system of $E$ with respect to $\omega(p, q ; z)$. Since

$$
\begin{aligned}
\log A(z) & =\lim \frac{1}{n(n+1)} \log A_{n}(z)=\lim \frac{2}{n(n+1)} \sum_{0 \leqslant j<k \leqslant n} \log \frac{1}{\omega\left(q_{i}, q_{k} ; z\right)} \\
& =\log \frac{1}{V(E, \omega(p, q ; z))}
\end{aligned}
$$

it follows from lemma 1 that $\log A(z)=I\left(\mu_{z}\right)$ where $\mu_{z}$ is the equilibrium measure with respect to $\omega(p, q ; z)$ (see p. 59).

Let

$$
u_{z}(p) \stackrel{\mathrm{df}}{=} \int_{E} \log \frac{1}{\omega(p, q ; z)} d \mu_{z}(q)
$$

Then

$$
u_{z}(p)=\int_{E^{*}} \log \frac{1}{\omega(p, q ; z)} d \mu_{z}(q)
$$

where $E^{*}$ is the kernel of $\mu_{z}$, i.e. the set of the points of $E$ such that, for every $z_{0} \in E^{*}$ and every open set $U_{0}$ containing $z_{0}, \mu_{z}\left(E \cap U_{0}\right)>0$. 
It is easy to see that

$$
\lim _{p \rightarrow z} u_{z}(p)=-\infty, \quad \lim _{p \rightarrow \infty} u_{z}(p)=-\infty
$$

and

$$
u_{z}(p)=\int_{E^{*}} \log \frac{1}{|p-q|} d \mu_{z}(q)-\frac{1}{2} \log \frac{1}{|z-p|}-\frac{1}{2} \int_{E^{*}} \log \frac{1}{|z-q|} d \mu_{z}(q)
$$

From (3.1) and (3.2) it follows that $u_{\hat{\imath}}(p)$ is a function of $p$, sub. harmonic on the outside of $E$. Hence we can prove by the same method as in [1] or in [6] that $u_{z}(p)$ satisfies the maximum principle, i.e. if $u_{z}(p) \leqslant K$ for $p \in E$, where $K$ is a constant, then $u_{z}(p) \leqslant K$ for every $p$. Hence

$$
u_{z}(p) \leqslant \sup _{q \in E^{*}} u_{z}(q)
$$

for every $p$.

Lemma 2. For every $p \in E$ except a set whose capacity with respect to $|p-q|$ equals aero we have the inequality

$$
u_{z}(p) \geqslant \sup _{q \in E^{*}} u_{z}(q)
$$

Proof. In the opposite case there would exist a set $F \subset E$ such that $C(F,|p-q|)>0$ and

$$
u_{z}(p)<\sup _{q \in E^{*}} u_{z}(q) \quad \text { for } \quad p \in F
$$

Hence there would exist a positive number $\varepsilon$ and a closed subset $F_{0}$ of $F$ with a positive capacity such that

$$
u_{z}(p)<\sup _{q \in E^{*}} u_{z}(q)-2 \varepsilon \quad \text { for } \quad p \in F_{0} .
$$

Since $z$ does not belong to $E$, it is easy to prove that also $C\left(F_{0}, \omega(p, q ; z)\right)$ $>0$. It is evident that there exists a point $p_{0}$ belonging to $E^{*}$ such that

$$
u_{z}\left(p_{0}\right)>\sup _{q \in E^{*}} u_{z}(q)-\varepsilon
$$

The potential $u_{z}(p)$ for a fixed $z$ is the lower semi-continuous function of $p$. Hence and from (3.6) it follows that there exists a neighbourhood $U_{0}$ of the point $p_{0}$ such that

$$
u_{\varepsilon}(p)+\varepsilon>\sup _{q \in E^{*}} u_{z}(q) \quad \text { for } \quad p \in U_{0} .
$$

Diminishing $U_{0}$ if necessary, we can suppose that the distance of $\boldsymbol{F}_{0}$ and $U_{0}$ is positive. Because $p_{0} \in E^{*}, \mu_{z}\left(U_{0}\right)>0$. Let $m \stackrel{\text { df }}{=} \mu_{z}\left(U_{0}\right)$. Since $C\left(F_{0}, \omega(p, q ; z)\right)>0$, there exists (see p. 59) a non-negative measure $\sigma^{\prime}(e)$ 
defined on the Borel subsets of $F_{0}$ such that

$$
\int_{F_{0}}\left[\int_{F_{0}} \log \frac{1}{\omega(p, q ; z)} d \sigma^{\prime}(q)\right] d \sigma^{\prime}(p)<\infty, \quad \sigma^{\prime}\left(F_{0}\right)=1
$$

Let $\sigma^{\prime \prime}(e)=m \sigma^{\prime}(e)$. Since

$$
\int_{F_{0}}\left[\int_{F_{0}} \log \frac{1}{\omega(p, q ; z)} d \sigma^{\prime \prime}(q)\right] d \sigma^{\prime \prime}(p)=m^{2} \int_{F_{0}}\left[\int_{F_{0}} \log \frac{1}{\omega(p, q ; z)} d \sigma^{\prime}(q)\right] d \sigma^{\prime}(p)
$$

we have by (3.7)

$$
\int_{F_{0}}\left[\int_{F_{0}} \log \frac{1}{\omega(p, q ; z)} d \sigma^{\prime \prime}(q)\right] d \sigma^{\prime \prime}(p)<\infty, \quad \sigma^{\prime \prime}\left(F_{0}\right)=m .
$$

Let us consider the measure $\sigma(e)$ defined as follows:

$$
\sigma(e)= \begin{cases}-\mu_{z}(e) & \text { for the Borel sets } e \subset U_{0}, \\ \sigma^{\prime \prime}(e) & \text { for the Borel sets } e \subset F_{0}, \\ 0 & \text { for the Borel sets lying in the outside } U_{0} \text { and } F_{0} .\end{cases}
$$

Let $\varrho$ be a positive number smaller than 1 . It is evident that the measure $\mu_{z}+\varrho \sigma$ belongs to $M$. By the same method as in [1] and [6] we can prove that

$$
I(\sigma)<\infty
$$

and

$$
I\left(\mu_{z}+\varrho \sigma\right)-I\left(\mu_{z}\right) \leqslant-\varrho[2 m \varepsilon-\varrho I(\sigma)] \text {. }
$$

For sufficiently small $\varrho$ the right side of (3.9) is, by (3.8), smaller than zero; so $\mu_{z}$ would not realise the infimum of the integrals $I(\mu)$, which is a contradiction of the definition of $\mu_{z}$. The lemma is thus proved.

From (3.3) and (3.4) we have the equality

$$
u_{z}(p)=\sup _{q \in E^{*}} u_{z}(q)
$$

for $p \in E$ except a set of capacity zero (for example, with respect to $|p-q|$ ).

We shall introduce the following notations:

$$
\begin{gathered}
h(p, z) \stackrel{\mathrm{d} f}{=} \int_{E} \log \frac{1}{|p-q|} d \mu_{z}(q)-\frac{1}{2} \log \frac{1}{|p-z|}, \\
\gamma(z)=\sup _{p \in E^{*}} u_{z}(p)+\frac{1}{2} \int_{E^{*}} \log \frac{1}{|q-z|} d \mu_{z}(q) .
\end{gathered}
$$

From (3.2) and (3.10) it follows that

$$
h(p, z)=\gamma(z)
$$


for $p \in E$ except a set of capacity zero. The function $h(p, z)$ considered as a function of $p$ on $E$ is continuous except a set of capacity zero. So we can prove by the same method as in [1] and [6] that the function $h(p, z)$ is continuous in the whole plane except a set of capacity zero.

Let $\mathrm{C} E$ denote the complementary set of $E$. $\mathrm{C} E$ consists of an at most enumerably infinite number of domains. Let $D_{\infty}$ be that one of those domains which contains $z=\infty$ and let $F_{\infty}$ be its boundary. Let $C(E)$ be the capacity of $E$ with respect to $|p-q|$.

We shall prove

Lemma 3. If $z_{0} \in E$ and $z_{0}$ is a regular point for $D_{\infty}$ with respect to the Dirichlet problem, then

$$
\gamma(z) \rightarrow \frac{1}{2} \log \frac{1}{C(E)} \quad \text { as } \quad z \rightarrow z_{0} \quad\left(z \in D_{\infty}\right) .
$$

Proof. Let $\mu$ be an equilibrium measure with respect to the generating function $|p-q|$. It is known (see [1], [6]) that for every $z \epsilon E$ except a set of capacity zero we have the equality

$$
u(z)=\int_{E} \log \frac{1}{|p-z|} d \mu(p)=\log \frac{1}{C(E)}
$$

and for every $z$

$$
u(z) \leqslant \log \frac{1}{C(E)} .
$$

In [1] and [6] it is proved that if $\int_{E} \log \frac{1}{|p-z|} d \mu(z)<\infty$ and $C\left(E_{0}\right)=0$ then $\mu\left(E_{0}\right)=0$. Hence and by the Fubini theorem we have

$$
\begin{aligned}
\gamma(z) & =\int_{E} \gamma(z) d \mu(p)=\int_{E} h(p, z) d \mu(p) \\
& =\int_{E}\left[\int_{E} \log \frac{1}{|p-q|} d \mu_{z}(q)-\frac{1}{2} \log \frac{1}{|p-z|}\right] d \mu(p) \\
& =\int_{E}\left[\int_{E} \log \frac{1}{|p-q|} d \mu(p)\right] d \mu_{z}(q)-\frac{1}{2} \int_{E} \log \frac{1}{|p-z|} d \mu(p),
\end{aligned}
$$

and so by (3.12) we have

$$
\gamma(z)=\log \frac{1}{C(E)}-\frac{1}{2} u(z)
$$

Since (see [1], [6]) $u(z) \rightarrow \log \frac{1}{C(\bar{E})}$, as $z \rightarrow z_{0}$, it follows from (3.13) that $\gamma(z) \rightarrow \frac{1}{2} \log \frac{1}{C(E)}$ as $z \rightarrow z_{0}$, q.e.d. 
By (3.11), (3.12), (3.13) and (3.14) it is easy to prove that the function

$$
\begin{aligned}
G(p, z) & =-2 h(p, z)+2 \gamma(z)+u(p)-\log \frac{1}{C(E)} \\
& =\log \frac{1}{|p-z|}-2 \int_{E} \log \frac{1}{|p-q|} d \mu_{z}(q)+\log \frac{1}{C(E)}-u(z)+u(p)
\end{aligned}
$$

is the Green function for the domain $D_{\infty}$ with the pole at $z$.

4. A certain property of the Green function. Let $G(p, z)$ be the Green function for $D_{\infty}$ (defined in Chapter 3 ) with the pole at $z$. We shall always assume that $z \neq \infty$.

It is known that there exists a finite limit

$$
\lim _{p \rightarrow z, p \neq z}\left[G(p, z)-\log \frac{1}{|p-z|}\right]
$$

and the function $U(p, z)$ defined for $z, p \in D_{\infty}$ by the formulas

$$
U(p, z)=\left\{\begin{array}{lll}
G(p, z)-\log \frac{1}{|p-z|} & \text { for } & p \neq z, \\
\lim _{p \rightarrow z, p \neq z}\left[G(p, z)-\log \frac{1}{|p-z|}\right] & \text { for } & p=z
\end{array}\right.
$$

is the solution of the Dirichlet problem for $D_{\infty}$ with the boundary value equal to $--\log \frac{1}{|p-z|}$ on $F_{\infty}$.

About the function $U(p, z)$ we shall prove the following

LEMMA 4. If $z_{0} \in F_{\infty}$ and $z_{0}$ is a regular point for the Dirichlet problem, then $\lim _{z \rightarrow z_{0}, z \in D_{\infty}} U(z, z)=-\infty$.

Proof. Let $K$ be an arbitrary positive number. Let

$$
\log _{K} x=\left\{\begin{array}{rll}
\ln x & \text { for } & 0<x \leqslant e^{K} \\
K & \text { for } & x>e^{K}
\end{array}\right.
$$

and let $U_{K}(p, z)$ be the solution of the Dirichlet problem for $D_{\infty}$ with the bonndary value equal to $-\log _{K} \frac{1}{|p-z|}$. We put $\log _{K} \frac{1}{|p-z|} \stackrel{\text { df }}{=} K$ for $p=z$. It is easy to see that

$$
\lim _{z \rightarrow z_{0}, z \in D_{\infty}} \log _{K} \frac{1}{|p-z|}=\log _{K} \frac{1}{\left|p-z_{0}\right|} \quad\left(z_{0} \in F_{\infty}\right)
$$

and the convergence is uniform with respect to $p \in D_{\infty}$. Hence there exists a $\delta>0$ such that for every $p \in F_{\sim}$

$$
\left|\log _{K} \frac{1}{|p-z|}-\log _{K} \frac{1}{\left|p-z_{0}\right|}\right|<1 \text {, when } \quad\left|z-z_{0}\right|<\delta, z \in D_{\infty} \text {. }
$$


From the last inequality and from the maximum principle for harmonic functions we have

$$
\left|U_{K}(p, z)-U_{K}\left(p, z_{0}\right)\right|<1, \quad \text { as } \quad\left|z-z_{0}\right|<\delta, p, z \in D_{\infty} .
$$

Putting $p=z$ we shall obtain

$$
U_{K}(z, z)<1+U_{K}\left(z, z_{0}\right), \quad \text { as } \quad\left|z-z_{0}\right|<\delta, z \in D_{\infty}
$$

and hence

$$
U(z, z)<1+U_{K}\left(z, z_{0}\right) \quad \text { for } \quad\left|z-z_{0}\right|<\delta, z \in D_{\infty}
$$

(because $U(p, z) \leqslant U_{K}(p, z)$ ). Since $z_{0}$ is a regular point, we have

$$
\lim _{z \rightarrow z_{0}, z \in D_{\infty}} U_{K}\left(z, z_{0}\right)=U_{K}\left(z_{0}, z_{0}\right)=-K .
$$

Hence and from (4.5) it follows that $\varlimsup_{z \rightarrow z_{0}, z \in D_{\infty}} U(z, z) \leqslant 1-K$ and, since $K$ is arbitrary positive number, we have $\lim _{z \rightarrow z_{0}, z \in D_{\infty}} U(z, z)=-\infty$, q.e.d.

5. Proof of the Inequality $A(z)<C(z)$. Let $D_{\infty}$ be the domain defined in Chapter 4 and $z \in D_{\infty}$.

Theorem 1. If the capacity $C(E)$ of $E$ is positive then, for every $z \in D_{\infty}$, $z \neq \infty, A(z)<C(z)\left({ }^{1}\right)$.

Proof. From the definition of $A(z)$ and $B(z)$ it follows that $A(\infty)$ $=B(\infty)$ and for every $z$

$$
A(z) \leqslant C(z) .
$$

The function $\frac{1}{n(n+1)} \log A_{n}(z)$ is the lower envelope of the harmonic functions $\frac{1}{n(n+1)} \sum_{j \neq k} \log \left|\frac{z-p_{k}}{p_{j}-p_{k}}\right|$. Hence (see p. 66) $\log A(z)$ is a superharmonic function in $D_{\infty}-\{\infty\}$. Since the function $\log C(z)$ (see [5]) is the Green function for $D_{\infty}$ with the pole at infinity, it follows from (5.1) that $\log A(z)-\log C(z)$ is a non-positive superharmonic function in $D_{\infty}-\{\infty\}$; thus in order to prove our theorem it is sufficient to prove that there exists one point $z^{\prime} \in D_{\infty}$ such that $A\left(z^{\prime}\right)<C\left(z^{\prime}\right)$.

Let $z_{0}$ be a regular point for $D_{\infty}$. Since

$$
G(p, z)=\log \frac{1}{|p-z|}-2 \int_{E} \log \frac{1}{|p-q|} d \mu_{z}(q)+\log \frac{1}{C(\bar{E})}-u(z)+u(p)
$$

(1) Editor's remark: J. Siciak has observed that there exist sets $E$ such that the inequality $A(z)<C(z)$ holds not for all points $z \in D_{\infty}(z \neq \infty)$. 
is the Green function for $D_{\infty}$ with the pole in $z$ (see (3.14)) we have (see $(4.2)$ )

$$
U(z, z)=-2 \int_{E} \log \frac{1}{|z-q|} d \mu_{z}(q)+\log \frac{1}{C(E)}
$$

Hence and from lemma 4 it follows that

$$
\int_{E} \log \frac{1}{|q-z|} d \mu_{z}(q) \rightarrow \infty, \quad \text { as } \quad z \rightarrow z_{0}\left(z \in D_{\infty}\right)
$$

Since

$$
\log A(z)=I\left(\mu_{z}\right)=\int_{E} h(p, z) d \mu_{z}(p)-\frac{1}{2} \int_{E} \log \frac{1}{|p-z|} d \mu_{z}(p)
$$

(see p. 66 and p. 68), from (3.11) using the same method as in the proof of lemma 3 we shall obtain

$$
\log A(z)=\gamma(z)-\frac{1}{2} \int_{E} \log \frac{1}{|p-z|} d \mu_{z}(p) .
$$

From (5.2), (5.3) and lemma 3 it follows that $\log A(z) \rightarrow \infty$ as $z \rightarrow z_{0}\left(z \in D_{\infty}\right)$. Since $\log C(z)$ is the Green function it follows that $\log C(z) \rightarrow 0$ as $z \rightarrow z_{0}$ $\left(z \in D_{\infty}\right)$. Hence there exists a $z^{\prime} \in D_{\infty}$ such that $A\left(z^{\prime}\right)<C\left(z^{\prime}\right)$, q.e.d.

EXAMPLE. From (3.12) it follows that $\log \frac{1}{C(E)}-u(z)$ is the Green function for $D_{\infty}$ with the pole $z=\infty$, and so $\log C(z)=\log 1 / C(E)-u(z)$.

Let $E$ be the circle $|z|=1$. In this case (see [5], pp. 270 and 281) $C(E)=C(E,|p-q|)=V(E,|p-q|)=1, \log C(z)=\log |z|$ for $z \in D_{\infty}$ and $\log \left|\frac{p \bar{z}-1}{p-z}\right|$ is the Green function for $D_{\infty}$ with the pole at $z$. Hence and from (3.14) it follows that

$$
\log \left|\frac{p \vec{z}-1}{p-z}\right|=\log \frac{1}{|p-z|}-2 \int_{E} \log \frac{1}{|p-q|} d \mu_{z}(q)-\log \frac{1}{|z|}+\log \frac{1}{|p|}
$$

and hence

$$
\log |p \bar{z}-1|=-2 \int_{\bar{E}} \log \frac{1}{|p-q|} d \mu_{z}(q)+\log |z|-\log |p| .
$$

If $p \rightarrow z$ then from (5.4) we shall obtain

$$
\left.\log || z\right|^{2}-1 \mid=-2 \int_{E} \log \frac{1}{|z-q|} d \mu_{z}(q)
$$


Since (see (5.3) and (3.13))

$$
\begin{aligned}
\log A(z) & =\gamma(z)-\frac{1}{2} \log \frac{1}{|z-q|} d \mu_{z}(q) \\
& =\log \frac{1}{C(E)}-\frac{1}{2} u(z)-\frac{1}{2} \int_{E} \log \frac{1}{|z-q|} d \mu_{z}(q) \\
& =\frac{1}{2} \log |z|-\frac{1}{2} \int_{E} \log \frac{1}{|z-q|} d \mu_{z}(q),
\end{aligned}
$$

we have by (5.5)

$$
\log A(z)=\frac{1}{2} \log |z|+\left.\frac{1}{4} \log || z\right|^{2}-1|=\log |^{4}\left[z_{i}^{|2|}\left|\overline{|z|^{2}} \overline{-1}\right|,\right.
$$

i.e. $A(z)=\sqrt[4]{\left.|z|^{2}|| z\right|^{2}-1 \mid}$ for $z \in D_{\infty}$.

In this case it is easy to see that $A(z)=\bar{l}^{\prime}\left|\overline{\left.z\right|^{2}}\right|\left|\overline{z_{1}^{2}-1}\right|<|z|=C(z)$ for $|z| \geqslant 1$, i.e. for $z \in D_{\infty}$.

Remark 1. It is known that if the set $E$ is the circle $|z|=1$ then $C(z)=1$ for $|z| \leqslant 1$. It is also easy to prove that $A(z)=\mathbf{V}^{\prime} /|z|^{2}-1 \mid$ for $|z| \leqslant 1$.

Remark 2. From the definition of $A(z)$ and $C(z)$ it follows that $\log A(z)-\log C(z) \rightarrow 0$ as $z \rightarrow \infty$. Hence and from the inequality $A(z)<C(z)$ for $z \in D_{\infty}, z \neq \infty$ it follows that $\log A(z)$ cannot be a harmonic function.

6. Proof of the equality $C(z)=D(z)$.

Theorem 2. If $V(E,|p-q|)>0$ then $C(z)=D(z)$ for every $z$ which does not belong to $E$.

Proof. At first we shall prove that

$$
\log D(z) \leqslant \log C(z) \quad \text { for } \quad z \in D_{\infty} .
$$

Let

$$
q^{n}=\left\{q_{0}, q_{1}, \ldots, q_{n}\right\}
$$

be the $n$th extremal system with respect to the generating function $\omega^{\prime}(p, q ; z)=\frac{|p-q|}{|z-p||z-q|}$. Let us denote by $\mu_{z}^{n}(e)$ the measure defined on the Borel subsets of $E$ as follows:

$$
\mu_{z}^{n}(e)=\frac{k}{n},
$$

where $k$ is the number of the points of the $n$th extremal system (6.2) contained in $e$. From the sequence (6.3) we can choose (see [1]) a subsequence $\mu_{z}^{n_{k}}(e)$ convergent to a measure $\mu_{z}^{\prime}(e)$ :

$$
\mu_{z}^{n_{k}}(e) \rightarrow \mu_{z}^{\prime}(e) \quad \text { as } \quad k \rightarrow \infty .
$$


From the proof of lemma 1 it is easy to deduce that the measure $\mu_{z}^{\prime}(e)$ realises the infimum of the integrals

$$
I(\mu)=\int_{E}\left[\int_{E} \log \frac{1}{\omega^{\prime}(p, q ; z)} d \mu(q)\right] d \mu(p)
$$

for $\mu \in M$ (see p. 58 ).

Let us introduce the notation

$$
\begin{gathered}
u_{z}^{\prime}(p) \stackrel{\text { df }}{=} \int_{E} \log \frac{1}{\omega^{\prime}(p, q ; z)} d \mu_{z}^{\prime}(q), \\
h^{\prime}(p, z) \stackrel{\text { df }}{=} \int_{E} \log \frac{1}{|p-q|} d \mu_{z}^{\prime}(q)-\log \frac{1}{|p-z|}, \quad \gamma^{\prime}(z) \stackrel{\text { df }}{=} \sup _{p \in E^{*}} h^{\prime}(p, z),
\end{gathered}
$$

where $E^{*}$ is the kernel of $E$. It can be proved by the same method as in Chapter 3 that

$$
I\left(\mu_{z}^{\prime}\right)=\gamma^{\prime}(z)=-\int_{\dot{E}} \log \frac{1}{|p-q|} d \mu_{z}^{\prime}(q)
$$

and

$$
\gamma^{\prime}(z)=\log \frac{1}{C(E)}-u(z)
$$

where $C(E)=C(E,|p-q|)$ is the capacity and $u(z)$ is the equilibrium potential with respect to the generating function $|p-q|$. By the same method as in [1] and [6] we can prove also that

$$
u_{z}(p) \leqslant I\left(\mu_{z}^{\prime}\right)
$$

for every $p$. From (3.12) and (3.13) it follows that $\gamma^{\prime}(z)$ is the Green function for $D_{\infty}$ with the pole at $\infty$. In order to prove (6.1) it is sufficient to prove the inequality

$$
\log D(z) \leqslant \gamma^{\prime}(z) \text { for } \quad z \epsilon D_{\infty}
$$

Let us write

$$
D_{n}^{(j)}(z) \stackrel{\text { de }}{=} \prod_{\substack{k=0 \\ k \neq j}}^{n}\left|\frac{z-q_{j}}{q_{k}-q_{j}}\right|, \quad j=0,1, \ldots, n .
$$

Without any loss of generality we can assume that $D_{n}^{(0)}(z) \geqslant D_{n}^{(1)}(z) \geqslant \ldots$ $\ldots \geqslant D_{n}^{(n)}(z)$. It is evident that

$$
D_{n}(z) \leqslant D_{n}^{(0)}(z),
$$


where $D_{n}(z)$ is defined on page $5 \pi$. Let

$$
\mathcal{J}_{n}^{(0)}(z) \stackrel{\mathrm{d} f}{=} \prod_{k=1}^{n} \omega^{\prime}\left(q_{0}, q_{k} ; z\right)=\frac{1}{D_{n}^{(0)}(z)} \prod_{k=1}^{n}\left|z-q_{k}\right| .
$$

Since system (6.2) is the extremal system, we can prove by the same method as in $[5]$, p. 267 that

$$
\Delta_{n}^{(0)}(z)=\prod_{k=1}^{n} \omega^{\prime}\left(q_{0}, q_{k} ; z\right)=\sup _{p \in E} \prod_{k=1}^{n} \omega^{\prime}\left(p, q_{k} ; z\right) .
$$

Hence

$$
\frac{1}{n} \sum_{k=1}^{n} \log \frac{1}{\omega^{\prime}\left(p, q q_{k} ; z\right)} \geqslant \log \frac{1}{\sqrt[n]{\Delta_{n}^{(0)}(z)}} \quad \text { for every } p \in E .
$$

Integrating the last inequality with respect to $\mu_{z}^{\prime}(e)$ we shall obtain (see p. 61)

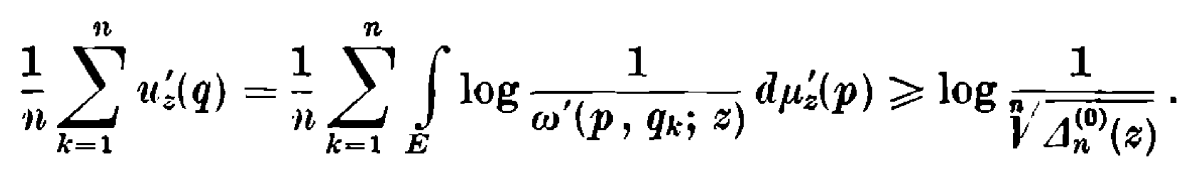

Hence and from inequality (6.8) we shall obtain

$$
I\left(\mu_{z}^{\prime}\right) \geqslant \log \frac{1}{\sqrt[n]{\Delta_{n}^{(0)}(z)}} .
$$

By (6.10), (6.11) and (6.12) we have

In particular

$$
I\left(\mu_{z}^{\prime}\right) \geqslant \frac{1}{n} \log D_{n}(z)-\frac{1}{n} \sum_{k=1}^{n} \log \frac{1}{\left|z-q_{k}\right|} .
$$

$$
I\left(\mu_{z}^{\prime}\right) \geqslant \frac{1}{n_{k}} \log D_{n_{k}}(z)-\frac{1}{n_{k}} \sum_{l=1}^{n_{k}} \log \frac{1}{\left|z-q_{l}\right|}
$$

(see p. 7t). Hence we have

$$
I\left(\mu_{z}^{\prime}\right) \geqslant \log D(z)-\int_{E} \log \frac{1}{|p-z|} d \mu_{z}^{\prime}(p) \quad \text { or } \quad \gamma^{\prime}(z) \geqslant \log D(z),
$$

i.e. inequality (6.1).

In order to prove that $\log C(z)=\log D(z)$ for $z \in D_{\infty}$ it is sufficient to prove that

$$
\gamma^{\prime}(z) \leqslant \log D(z) \text { for } \quad z \in D_{\infty}
$$


Let $r^{(n)}=\left\{r_{0}, r_{1}, \ldots, r_{n}\right\}$ be the $n$th system of points realising the infimum of $D_{n}\left(z ; p^{(n)}\right)$ and let $\mu_{z}^{\prime \prime}$ be a measure defined similarly to the measure $\mu_{z}^{\prime}$ (see p. 73). It is easy to prove (see the definition of $D(z)$; that for every $p \in E$

$$
\log D(z) \geqslant \int_{E} \log \frac{1}{|p-q|} d \mu_{z}^{\prime \prime}(q)-\log \frac{1}{|p-z|} .
$$

Let $\mu$ be $n$th extremal measure with respect to $|p-q|$. By the same method as in the proof of lemma 3 (see p. 69) we can prove that

$$
\begin{aligned}
\log D(z) & =\int_{E} \log D(z) d \mu(p) \geqslant \int_{E}\left[\int_{\dot{E}} \log \frac{1}{|p-q|} d \mu_{z}^{\prime \prime}(q)-\log \frac{1}{|p-z|}\right] d \mu(p) \\
& =\log \frac{1}{C(E)}-u(z)=\gamma^{\prime}(z)
\end{aligned}
$$

i.e. inequality (6.14).

In the case where $z$ does not belong to $D_{\infty} \cup F_{\infty}$ we have (see [5], p. 278) $\log C(z)=0$ and (see [1], [6]) $u(z)=\log \frac{1}{C(E)}$. We can also prove by the same method as in the case of $z \in D_{\infty}$ that $\log D(z)=0$.

Remark. It is easy to prove that if $z \in F_{\infty}$ and $z$ is a regular point for the Dirichlet problem for $D_{\infty}$ then $D(z)=C(z)=1$. If $z$ is a irregular point then $D(z)$ can be greater than $C(z)$.

EXAmPLE. Let $E$ be the set consisting of the circle $|z|=1$ and the point $z=5$. In this case $C(\check{5})=1$ (see [5], p. 278) and

Hence $D(5) \geqslant 2>1=C(5)$.

$$
\left.D_{n}(5)=\inf _{p^{(n)}} \mid \max _{\substack {(j) \\
\begin{subarray}{c}{k=0 \\
k=j{ ( j ) \\
\begin{subarray} { c } { k = 0 \\
k = j } }\end{subarray}}^{n} \frac{\left|5-p_{j}\right|}{\left|p_{k}-p_{j}\right|} \geqslant \frac{4^{n}}{2^{n} \cdot 6}\right\} \text {. }
$$

\section{References}

[1] 0. Frostman, Potentiel d'équilibre et capacité des ensembles, Thèse, Lund 1935.

[2] F. Leja, Sur les suites les ensembles fermés et la fonction de Green, Ann. Soc.

Pol. Math. 12 (1958), pp. 57-71.

[3] - Sur certaines limites relatives aux polynômes de Lagrange et aux ensembles fermés, Bull. Acad. Pol. Sc. Lettr. Serie A (1933), pp. 281-289.

[4] - Sur une suite de fonctions liée aux ensembles plans fermés, Ann. Soc. Pol. Math. 13 (1934), pp. 55-58.

[5] - Teoria funkcji analitycznych, Warszawa 1957.

[6] M. Tsuji, Fundamental theorems in the potential theory, Journ. Math. Soc. Jap. 4 (1952), pp. 70.96. 